\title{
Potentiale datengestützter Methoden zur Gestaltung und Optimierung mechanischer Fügeverbindungen
}

\section{Potentials of data-based methods for the design and optimization of mechanical joints}

\author{
Christoph Zirngibl ${ }^{1 *}$, Benjamin Schleich ${ }^{1}$, Sandro Wartzack ${ }^{1}$ \\ ${ }^{1}$ Engineering Design, Friedrich-Alexander-Universität Erlangen-Nürnberg \\ ${ }^{*}$ Korrespondierender Autor: \\ Christoph Zirngibl \\ Lehrstuhl für Konstruktionstechnik (KTmfk) \\ Martensstraße 9 \\ 91058 Erlangen \\ Telefon: +49 911/530296618 \\ Mail: zirngibl@mfk.fau.de
}

\begin{abstract}
Due to the increasing requirements on lightweight constructions, the demands for efficient joining processes are constantly rising. Therefor cold forming processes provide a faster and less expensive alternative to established thermal joining methods. In order to guarantee the joining reliability, not only the selection of a suitable process, but also the design and dimensioning of the joint is crucial. As a possible solution, data-driven methods offer procedures for the structuring of data and the targeted analysis of product features and key variables. Motivated by this, the paper shows a literature-based approach by using the example of clinching to illustrate the potentials and limits of data-based methods for the design and optimization of mechanical joining processes.
\end{abstract}

\section{Keywords}

Clinching, Data-driven optimization, Multiobjective Optimization 


\section{Einleitung}

Mechanische Fügeverfahren haben unter anderem aufgrund der Möglichkeit des Fügens beschichteter Bauteile sowie der Erzeugung von Mischmetallverbindungen und dem damit einhergehenden Leichtbaupotential in den letzten Jahrzehnten einen festen Platz sowohl in Klein- als auch Großserienanwendungen erobert [1]. Für die Gewährleistung deren Fügesicherheit liegt der Fokus neben der Wahl eines passenden Fügeverfahrens vor allem auf der Auslegung und Dimensionierung der Fügeverbindung. Während der Konstrukteur hierzu im Bereich thermischer Verfahren, insbesondere Schweißverbindungen, auf eine große Auswahl an Auslegungs- und Gestaltungsgrundlagen zurückgreifen kann [2], basiert die Bewertung mechanischer Fügeverfahren überwiegend auf Basis von Expertenwissen und weniger experimenteller Versuche [3, 4]. Resultierend ergeben sich für eine optimale Auslegung einer mechanischen Fügeverbindung oftmals wiederkehrende sowie kosten- und zeitintensive Anpassungen an der Gestalt der Fügepartner oder den gewählten Fügeparametern. Als eine mögliche Lösung bieten unter anderem Methoden des maschinellen Lernens Verfahren zur Strukturierung von Daten sowie der zielgerichteten Analyse von Produktmerkmalen und Zielgrößen. Folglich beleuchtet dieser Beitrag am Beispiel des Clinchens die Einsatzpotentiale und Grenzen von datengestützten Methoden für die Auslegung, Dimensionierung und Optimierung mechanischer Fügeverbindungen.

\section{Hintergrund und Forschungsproblem}

\subsection{Clinchen als umformtechnisches, mechanisches Fügeverfahren}

Das exemplarisch untersuchte Clinchen zählt laut DIN 8593-5 zu den umformtechnischen Fügeverfahren. Die Verbindung einzelner Blech-, Rohr- oder Profilteile (zwei oder mehrere) basiert ausschließlich auf der mechanischen Erzeugung lokaler Kaltumformungen, wodurch auf eine Verwendung von Hilfsfügeteilen (beispielsweise Niete) beziehungsweise Zusatzwerkstoffen verzichtet werden kann [4]. Durch anfängliches partielles Durchsetzen und anschließendes Stauchen, Breiten sowie Fließpressen entsteht eine unlösbare kraft- und formschlüssige Verbindung zwischen den jeweiligen Fügeelementen [4, 5]. Ein wesentlicher Vorteil des Verfahrens ist hierbei der Einsatz im Leichtbau. Speziell die Möglichkeit zur Variation unterschiedlicher Werkstoffe (v.a. Verbundwerkstoffe) beziehungsweise das Fügen von wenig schweißgeeigneten Materialien hat zu einer zunehmenden Anwendung von formund kraftschlüssigen Verbindungen geführt. Ebenso bietet das Clinchen die Möglichkeit zur Kombination beschichteter Bauteile bei gleichzeitigem Wegfall von mechanischen Vor- und Nacharbeiten. Eine robuste Auslegung der Fertigungswerkzeuge resultiert zusätzlich in einer Senkung der nötigen Wartungskosten. Zu den Einsatzgebieten des Verfahrens zählen insbesondere die Automobilbranche (v.a. Karosseriebau) sowie der Klima- beziehungsweise Lüftungsbau. [5, 6] Zu den qualitätsrelevanten Kenngrößen der Fügeverbindung gehören unter anderem die Ausprägung eines Hinterschnitts zwischen dem matrizen- und stempelseitigem Fügeelement, sowie der minimalste horizontale Halsdickenquerschnitt des stempelseitigen Fügeteils [6]. Für die Erzeugung und Optimierung dieser Eigenschaften, wurden bereits einige Forschungsarbeiten zur Relevanzanalyse ausgewählter Werkzeug- und Prozessparamter durchgeführt [u.a. 3, 10, 16]. Ausgehend von diesen soll für diesen Beitrag eine einheitliche Bezeichnung der Begrifflichkeiten Eigenschaften, Merkmale und Bauteilverhalten definiert werden. Hierbei kann zwischen den vom Produktentwickler direkt beeinflussbaren (Merkmale) und den nicht direkt zu bestimmenden (Eigenschaften) Kenngrößen unterschieden werden. Beispielsweise sind die in Bild 1 dargestellten geometrischen Kennwerte, speziell der Hinterschnitt und die Halsdicke, aus dem Fügeprozess hervorgehende Größen und können vom Konstrukteur nicht direkt gesteuert werden. Folglich sind diese den Produkteigenschaften zuzuordnen. Wiederum Parameter, welche die Ausformung des Hinterschnitts und der Halsdicke begünstigen, unter anderem Abmessungen der Fügewerkzeuge oder 
Umformgeschwindigkeiten, sind direkt vom Konstrukteur definierbar und somit als Produktmerkmale zu bezeichnen.

a)

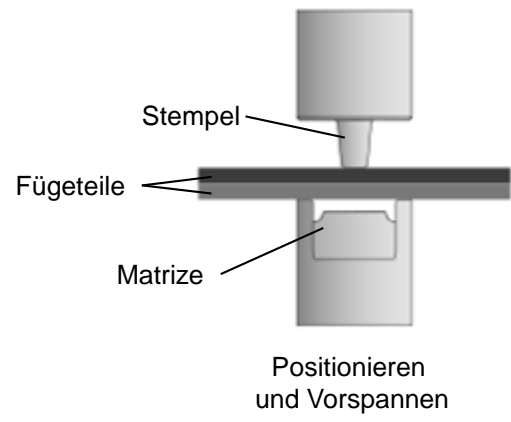

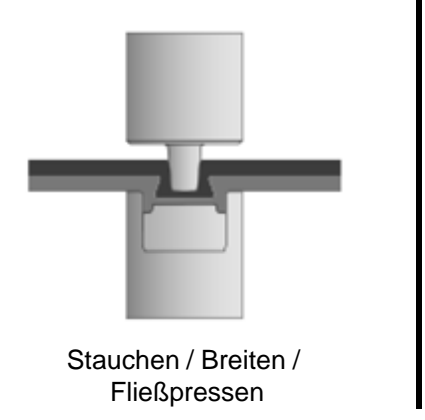

b)

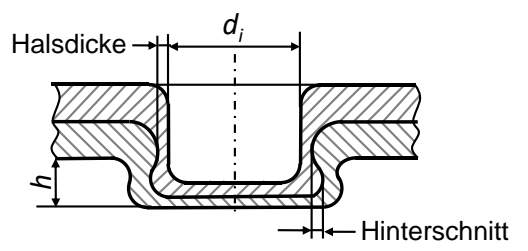

Bild 1: Verfahrensablauf eines Clinchprozesses (a) nach [7] sowie Beispiel geometrischer Kennwerte (b) nach [8].

Unter dem Verhalten des Bauteils (Clinchverbindung) können die resultierenden Festigkeitskennwerte (u.a. Zug- und Scherfestigkeiten) zusammengefasst werden. Deren Werte sind stark abhängig von der Ausprägung bestimmter Produkteigenschaften $[5,6]$.

\subsection{Forschungsproblem}

Für die analytische Betrachtung von Clinchverbindungen exisitieren bereits einige Forschungsarbeiten. Der Fokus bezieht sich deshalb zu Beginn dieses Beitrags auf der Bewertung dieser hinsichtlich der Vorgehensweisen zur Wissensentdeckung in Datenbanken (eng. Knowledge Discovery in Databases, KDD) und der daraus resultierenden Steigerung relevanter Auslegungskriterien. Ziel ist die Ausarbeitung einer Methoden-Matrix und die Beantwortung der Forschungsfrage, welche Einsatzpotentiale durch den Einsatz von datengestützen Methoden zur Analyse und Optmierung von mechanischen Fügeverfahren abgeleitet werden können. Dies soll am Beispiel des Clinchverfahrens aufgezeigt werden. Unter dem Begriff Optimierung ist hierbei die Identifizierung optimaler Parameterwerte (Werkzeug- und/oder Prozessparameter) für die Maximierung beziehungsweise Minimierung von relevanten Produkteigenschaften (zumeist Halsdicke, Hinterschnitt) zu verstehen. Somit sollen entsprechende datengestützte Methoden Extremwerte der einzelnen Zielfunktionen bestimmen und die dafür notwendigen Parameterkombinationen extrahieren. Unter datengestützten Methoden sind hierbei Vorgehensweisen zur Generierung einer Datenbasis, zur Metamodellierung und Klassifizierungen von Daten sowie der abschließenden Mehrzieloptimierung von Zielgrößen zu verstehen [9, 10]. Folglich soll durch die Analyse von Daten eine Betrachtung von Produkt- und Prozessmerkmalen (u.a. Stempeldurchmesser, Umformgeschwindigkeit) und deren Auswirkung auf ausgewählte Fügepunkteigenschaften erfolgen.

\section{Verwendete Methoden bzw. Vorgehensweisen}

Für die Identifizierung des aktuellen Stands der Technik wurde zu Beginn eine systematische Literaturrecherche durchgeführt. Dazu bildeten die Datenbanken Scopus, ScienceDirekt, Google Scholar und der Katalog der Universitätsbibliothek Erlangen-Nürnberg die Grundlage für die Ermittlung der vorhandenen Forschungsbeiträge. Um möglichst alle relevanten Informationen zur definierten Fragestellung zu erfassen, wurden die in Tabelle 1 dargestellten Begriffe mittels boolesche Operatoren verknüpft. Ebenso konnten irrelevante Beiträge durch die Anwendung von Sucheinschränkungen bereits im Vorhinein gefiltert werden. Hierzu zählten unter anderem der Suchzeitraum (Artikel der letzten 15 Jahre) sowie 
zentrale Fachbereiche (bspw. Engineering, Materials Science, etc.). Resultierend konnten 54 Beiträge ermittelt und in eine Datenbank überführt werden. Für die Bewertung der Relevanz der einzelnen Forschungsartikel, wurde beginnend eine Vorauswahl anhand der jeweiligen Titel vorgenommen und anschließend durch die Analyse der Zusammenfassungen/Abstracts eine finale Auswahl von 13 Beiträgen getroffen. Anknüpfend erfolgte eine Auswertung der Literaturdatenbasis hinsichtlich der Verwendung datengestützter Methoden bei der optimierten Auslegung und Dimensionierung von Clinchverbindungen. Die Ergebnisse sowie die Ableitung einer Methoden-Matrix ist in den folgenden Abschnitten erläutert.

Tabelle 1: Zerlegung der Forschungsfrage in einzelne Forschungsaspekte

\begin{tabular}{|c|c|c|c|c|}
\hline & Begriff 1 & Begriff 2 & Begriff 3 & Begriff 4 \\
\hline $\begin{array}{l}\text { Aspekte } \\
\text { Eng. }\end{array}$ & $\begin{array}{l}\text { „Datengestützte Methoden“ } \\
\text { Data-based method“ }\end{array}$ & $\begin{array}{l}\text { „Gestaltung“ } \\
\text { Design“ }\end{array}$ & $\begin{array}{l}\text { „Optimierung“ } \\
\text { Optimiz“ }\end{array}$ & $\begin{array}{l}\text { „Durchsetzfügen““ } \\
\text { Clinch* }\end{array}$ \\
\hline $\begin{array}{l}\text { Synonyme \& } \\
\text { Unterbegriffe }\end{array}$ & $\begin{array}{l}\text { Design of experiment } \\
\text { Response surface method* } \\
\text { Regression } \\
\text { Classification } \\
\text { Sensitivity analy* } \\
\text { Robust design* }\end{array}$ & $\begin{array}{l}\text { Implement* } \\
\text { Develop* } \\
\text { Dimension* } \\
\text { Model* }^{*}\end{array}$ & $\begin{array}{l}\text { Improve* } \\
\text { Optimis* } \\
\text { Genetic algorith* } \\
\text { Multi objective } \\
\text { optimi* }\end{array}$ & \\
\hline $\begin{array}{l}\text { Verwandte } \\
\text { Begriffe }\end{array}$ & $\begin{array}{l}\text { Data mining } \\
\text { Data analy* } \\
\text { Machine learning } \\
\text { Metamodel }^{*}\end{array}$ & & & $\begin{array}{l}\text { Clinching joint } \\
\text { Clinch join* }\end{array}$ \\
\hline
\end{tabular}

\section{Darstellung der aktuellen Forschungsarbeiten}

Dieser Abschnitt befasst sich mit der ausführlichen Darstellung der identifizierten Forschungsartikel bezüglich unterschiedlicher Herangehensweisen für die analytische Gestaltung von Clinchverbindungen.

In Oudjene et al. (2008) und Oudjene et al. (2009) wurde bereits, auf Basis einer simulationsbasierten Parameterstudie, die jeweiligen Auswirkungen unterschiedlicher Werkzeuggeometrien auf qualitätsrelevante Kenngrößen (hier: Halsdicke, Hinterschnitt) untersucht. Für die Beschreibung der Zusammenhänge zwischen einzelnen Einflussparametern wird nach der Response Surface Methode (RSM) vorgegangen. Ein statistischer Versuchsplan, basierend auf der Taguchi-Methode (drei Unterteilungsstufen für sechs Geometrieparameter), dient hierbei als Grundlage für die Generierung des benötigten Versuchsumfangs. Auf Basis einer Moving Least Squares Approximation konnte abschließend die Definition einer optimalen Werkzeugeometrie erzielt werden. Als Ergebnis zeigte sich eine Erhöhung der Zugfestigkeit der Clinchverbindung von $623 \mathrm{~N}$ auf $834 \mathrm{~N}$. [11, 12]

Lebaal et al. (2012) verfolgen in deren Beitrag das Ziel, auf Basis der Vorarbeiten von Oudjene et al. (2009), weitere Verbesserungen der Clinchverbindung zu erreichen. Für die Generierung von simulationsbasierten Daten wird in einem ersten Schritt ein statistischer Versuchsplan nach dem Central-Composite Design erzeugt. Resultierend bilden die aus einer 2D-Simulationen ermittelten Zielgrößen (Halsdicke, Hinterschnitt) die Grundlage für den anschließenden Optimierungsprozess. Dieser setzt sich aus den Vorgehensweisen der Response Surface Methode sowie der Einbeziehung einer Kriging Interpolationsmethode (Gaußprozess-Regressionen) zusammen. Folglich konnte eine Optimierung von vier augewählten Werkzeugparametern durchgeführt und eine Steigerung der Zugfestigkeit von 10\%, im Vergleich zu Oudjene et al. (2009), ermittelt werden. [12, 13] 
Lambiase et al. (2013) beschreiben ebenfalls ein Konzept zur Steigerung der Zugfestigkeit von Rundpunktclinchverbindungen. Beginnend wird eine Taguchi-L27-Methode für den Aufbau eines statistischen Versuchsplans (auf drei Leveln für fünf Geometrieparameter) erstellt. Die Haupteffekte der Werkzeugparameter auf die Zielgrößen werden anschließend durch ein künstliches neuronales Netz (engl. artificial neural network, ANN) beschrieben. Der Trainingsdatensatz umfasst hierbei alle 27 Versuchspunkte, wobei zwei Zwischendatensätze für die Validierung des ANN zurückgehalten werden. Mit einer Gesamtfehlerquote von $5 \%$ wird eine gute Übereinstimmung zwischen ANN-Vorhersagen und FE-Ergebnissen festgestellt. Eine Geometrieoptimierung konnte mittels genetischen Algorithmus erzielt werden. [14]

Ähnlich dem Vorgehen von Lebaal et al. (2012) betrachten Roux et al. (2013) die Optimierung der Werkzeuggeometrie mittels Gaußprozess-Regressionen. Ergänzend hierzu steht der Einfluss der duktilen Schädigung auf die Festigkeitseigenschaften der Fügeelemente im Mittelpunkt. Auf Basis von Sensitivitätsanalysen konnten prozessrelevante Parameter (u.a. Stempeldurchmesser und Matrizentiefe) identifiziert und eine Reduzierung des Gesamtparameterumfanges vorgenommen werden. Für die Festlegung optimaler Werkzeuggeometrien wurde ein EGO-Algorithmus in Kombination mit dem aus den Simulationsdaten generierten Kriging-Metamodellen verwendet. Somit konnte eine Erhöhung der Scherfestigkeit um 43\% und der Zugfestigkeit um 13\% erzielt werden. [15]

Grundlegende Erkenntnisse zur analytischen Bestimmung von Festigkeitsnachweisen bei Rundpunktclinchverbindungen konnte durch Drossel et al. (2013) und Israel (2015) erarbeitet werden. Deren Beiträge befassen sich mit der Anwendung von simulationsbasierten Sensitivitäts- und Robustheitsanalysen in Kombination mit der statistischen Versuchsplanung (Latin Hypercube Sampling). Die aufbauend auf Regressionsanalysen erzeugten Metamodelle liefern eine Möglichkeit zur Identifizierung von Zusammenhängen (lineare und nicht-lineare) relevanter Werkzeugparameter auf die resultierenden Produkteigenschaften. Diese bilden eine Grundlage für die anschließende Lösung eines Optimierungsproblems aus der simultanen Maximierung der Halsdicke und des Hinterschnitts. Nachfolgend konnte mittels der Adaptive Response Surface Methode und dem Aufbau einer Pareto-Front (ParetoOptimierung) eine Verbesserung der betrachteten Zielgrößen abgeleitet werden. [16, 17]

Wen et al. (2014) definieren in deren Beitrag eine Methode zur analytischen Betrachtung eines Nachbearbeitungsprozesses zur Reduzierung der Clinchpunktüberstandshöhe bei gleichzeitger Erhöhung der Festigkeit. Eine konventionell erzeugte Clinchverbindung unterliegt hierbei einem weiteren Umformungsschritt, durch welchen mittels entwickelter Konturwerkzeuge eine Modifizierung der ursprünglich generierten Geometrie vorgenommen werden kann. Ziel des Beitrages ist die optimierte Gestaltung der Werkzeuggeometrien für den Reshaping-Prozess. Hierbei bildet ein statistischer Versuchsplan, basierend auf der TaguchiMethode, die Grundlage für die numerische Versuchsdatengenerierung. Die anschließende Durchführung einer Varianzanalyse (ANOVA) ermöglicht die Kombination einzelner untersuchter Parameter zu einer optimierten Auslegung der Konturwerkzeuge. Resultierend konnte eine Reduzierung der Clichpunktüberstandshöhe von $1.7 \mathrm{~mm}$ auf $0.68 \mathrm{~mm}$ bei gleichzeitiger Steigerung der Zugfestigkeit von $230.8 \mathrm{~N}$ auf $331.4 \mathrm{~N}$ erzielt werden. [18]

Einen weiteren Beitrag zur simulationsbasierten Analyse und Gestaltung von Clinchverbindungen leisteten Eshtayeh et al. (2016) mit der Erweiterung des untersuchten Parameterraumes. Durch die Verwendung eines Taguchi-Versuchsplanes (drei Level) konnte ein Datenumfang von 27 Versuchspunkten für 11 Inputparameter erzeugt werden. Die Identifizierung statistisch relevanter Geometrieparameter wurde ähnlich dem Beitrag von Wen et al. (2014) mittels einer Varianzanalyse erzielt. Nach der Ermittlung der einflussreichsten Parameter, konnte durch die Anwendung einer Grey-basierten Taguchi-Methode optimale Parametersätze abgeleitet werden. Folglich wurden durch die Lösung des mehrkriteriellen Optimierungsproblemes (Kombination der bestmöglichen Parameterlevel) eine Steigerung der untersuchten Produkteigenschaften erreicht. [19] 
Han et al. (2016) untersuchten, ähnlich zu den Vorarbeiten von Lambiase et al. (2013), die optimierte Werkzeugauslegung zur Erzielung verbesserter Clinchpunktzugfestigkeiten. Für die simulationsbasierte Ermittlung dieser, wurde ein statistischer Versuchsplan (orthogonale Designmatrix auf drei Leveln) für vier Inputparameter generiert und neun Versuchpunkte abgeleitet. Ausgehend der Simulationsergebnisse konnte anschließend eine Bewertung der jeweiligen Parametersätze durchgeführt werden. Hierbei wurden für die einzelnen Parameterdesignlevel der orthogonalen Versuchsmatrix die jeweiligen Durchschnittswerte der erzielten Zugfestigkeiten der Clinchverbindung ermittelt. Resultierend konnte durch die Kombination der bestmöglichen Designlevel eine optimierte Auslegung der untersuchten Werkzeugparameter vorgenommen werden. [20]

In dem Beitrag von Wang, M. et al. (2018) wird eine Methode zur mathematischen Optimierung der Fügepunktfestigkeit mittels Bézier Kurven vorgestellt. Ziel ist die Beschreibung der Werkzeugkontur (Matrize) unabhängig zuvor ausgewählter Geometrieparameter. Beginnend wird für den Optimierungsprozess ein mathematisches Modell zur Definition des Mehrzielproblems aufgestellt. Die anschließende Bestimmung einer optimalen Kontur erfolgt durch die Kopplung eines genetischen Algorithmus mit den jeweiligen CAD/FEM-Modulen. Die direkte Übertragung von Designvariablen ermöglicht dadurch eine iterative Identifizierung wichtiger Konturbereiche und die stetige Optimierung dieser. Nach 700 Simulationen konnte ein bestmöglicher Werkzeugsatz abgeleitet werden, was zu einer Steigerung der Zug- und der Scherfestigkeit führte. [21]

Wang, X. et al. (2018) analysieren in deren Beitrag neben den Geometrie- ebenfalls die Einflüsse einzelner Prozessparameter. Als Vorgehensmethode wird ähnlich zu Lambiase et al. (2013) auf die Response Surface Methode zurückgegriffen. Dabei bildet ein Box-Behnken Design (neun Inputparameter, 130 Versuchspunkte) die Grundlage der anfänglichen Definiton des Versuchsumfangs. Für die Identifizierung von Wechselwirkungen wird eine Varianzanalyse durchgeführt. Folglich lassen sich lineare (Halsdicke) und quadratische Regressionsmodelle (Hinterschnitt, Zugfestigkeit) für die Quantifizierung des Einflusses der Inputparameter auf die Ausprägung der Produkteigenschaften beschreiben. Bei der Auswahl der optimierten Geometrie- und Prozessparameter wird das multikriterielle Optimierungsproblem durch den genetischen Algorithmus NSGA-II (non-dominated sorting genetic algorithm) gelöst. Anhand der resultierenden Pareto-Fronten können bestmögliche Lösungen für die Maximierung der relevanten Produkteigenschaften abgeleitet werden. [22]

Schwarz et al. (2020) versuchen die Identifizierung einer verbesserten Werkzeuggeometrie durch die Anwendung einer auf der Hauptkomponentenanalyse (engl. Principal component analysis, PCA) basierenden Optimierungsmethode. Ziel ist die Maximierung des Hinterschnittes und der Halsdicke unter Berücksichtigung der maximal erlaubten Normalspannung im Stempel. Die erzeugte Geometrie ist hierbei eine Rundpunktclinchverbindung für große Blechdicken (Gesamtblechdicke 5.0mm). Beginnend wird durch einen Advanced Latin Hypercube ein Versuchsumfang von 750 Punkte für 11 Inputparameter aufgebaut. Dieser bietet nachfolgend die Grundlage für die Durchführung einer PCA und der anschließenden Ableitung eines direkten funktionalen Zusammenhangs zwischen den Fügegeometrien und den analysierten Werkzeugkonturparametern. Auf Basis dieser Erkenntnisse können durch die Verwendung eines genetischen Algorithmus optimierte Parametereinstellungen abgeleitet werden. [23]

Für die konstruktive Gestaltung der Clinchverbindung existieren bereits einige Vorgaben in etablierten Standardwerken (bspw. Roloff/Matek). Unter anderem wird der Mindestabstand zwischen zwei Clinchverbindungen sowie die Anzahl an möglichen Punkten in linienförmiger Anordnung definiert [24]. Ebenso bestehen Grundregeln für die Werkstoffauswahl, das maximale Blechdickenverhältnis sowie die Anordnung von Blechen mit unterschiedlichen Werkstoffeigenschaften. Des Weiteren werden vereinzelte Anhaltswerte zu Verbindungsfestigkeiten (u.a. Scherzugkräfte) beispielhaft aufgeführter Clinchverbindungen angegeben und auf Mindestabmessungen geclinchter Bauteile hingewiesen. [4, 5, 6, 24] 
Eine zusammenfassende Darstellung sämtlicher Erkenntnisse ist in Form einer MethodenMatrix in Tabelle 2 am Ende des Beitrags abgebildet.

\section{Diskussion der Erkenntnisse}

In diesem Kapitel werden einzelne Methoden zur Wissensentdeckung in Datenbanken hinsichtlich ihrer Potentiale zur Auslegung, Dimensionierung und Optimierung von mechanischen Fügeverbindungen diskutiert. Hierfür bilden die zuvor erarbeiteten Erkenntnisse zur Analyse von Clinchverbindungen die Diskussionsgrundlage.

\subsection{Aufbau einer Datenbasis durch statistische Versuchsplanung}

Allgemein bietet die statistische Versuchsplanung eine effiziente Herangehensweise zur Planung und Auswertung von Versuchsreihen für die Analyse von Parametereinflüssen. Die gewählten Beschreibungsmodelle variieren hierbei in der Anzahl der notwendigen Stichprobengröße und deren Fähigkeit zur Darstellung komplexer Zusammenhänge. [25]

Interessante Ansätze für den Aufbau eines statistischen Versuchsplans mittels der TaguchiMethode zeigten bereits die Arbeiten von Oudjene et al. (2008) und Oudjene et al. (2009). Der resultierende dreistufige Versuchsplan ermöglicht, auf Basis geringer Versuchspunkte, eine gute Abschätzung der Effekte von Inputfaktoren auf die zu untersuchenden Clinchpunkteigenschaften. Die orthogonalen Felder begünstigen hierbei eine balancierte und gleichmäßige Gewichtung der jeweiligen Faktorstufen und die Analyse der einzelnen Versuchpunkte hinsichtlich derer Auswirkung auf die Zielgrößen (Halsdicke, Hinterschnitt). Der Einfluss von individuellen Faktoren hat somit keine Auswirkung auf die Bewertung sonstiger Merkmale [25]. Die gute Anwendbarkeit der Taguchi-Methode wird durch den Einsatz in weiteren Arbeiten von Lambiase et al. (2013), Wen et al. (2014) und Eshtayeh et al. (2016) demonstriert. Für die Durchführung von umfangreichen numerischen Simulationen zeigte ein statistischer Versuchsplan nach dem Latin Hypercube Design vielversprechende Resultate. Die Stärke dieser Methode ist die gute Analysemöglichkeit vieler Inputfaktoreinstellungen. Ein "over-fitting" in der Versuchsplanung ist somit nahezu ausgeschlossen [25]. Speziell in den Arbeiten von Drossel et al. (2013), Israel (2015) sowie Schwarz et al. (2020) konnten dadurch eine umfängliche Abdeckung des definierten Wertebereichs sämtlicher Inputparameter ermöglicht werden. Der zusätzliche Informationsgewinn über Zusammenhänge zwischen Inputparametern und Zielgrößen begünstigt somit die nachfolgende Datenanalyse [25].

\subsection{Methoden zur Wissensentdeckung in Datenbanken}

Für die Analyse von Daten ist es nötig auf Basis erzeugter Ersatzmodelle beziehungsweise strukturierter Vorgehensweisen ein ausreichendes Verständnis komplexer Zusammenhänge zu ermöglichen [25]. Hierfür zeigen unter anderem maschinelle Lernprozesse, wie sie beispielsweise in den Arbeiten von Roux et al. (2013) und Lambiase et al (2013) verwendet werden, ein hohes Potential für die Wiedergabe funktioneller Abhängigkeiten zwischen den Input- und Zielgrößen. Der Aufbau eines künstlichen neuronalen Netzes beziehungsweise die Verwendung von Gauß-Regressionsprozessen (Kriging) bieten eine flexible und selbstständige Anpassung an vorhandene Datenpunkte. Stellvertretend ist in beiden Beiträgen jedoch der sehr geringe Versuchumfang zu betrachten. Die meisten maschinellen Lernprozesse benötigen eine ausreichende Datenmenge um hinreichend zu funktionieren [9, 25]. Beispielsweise erzielt das ANN in [14] zwar im ersten Schritt eine gute Beschreibung der Parameterzusammenhänge (Fehlerquote $5 \%$ ), jedoch zeigte sich bei nachfolgenden Durchläufen teilweise eine Verschlechterung der Vorhersagequalität (Fehlerquote 15\%). Eine weitere Herangehensweise zur Analyse von Daten bietet, unter anderem in den Arbeiten von Oudjene et al. (2009) und Wang, X. et al. (2018), die Anwendung der Response Surface 
Methode. Diese ermöglicht eine Beschreibung von Effekten und Zusammenhängen betrachteter Parameter auf Basis einer Generierung von Regressionspolynomen. Bei einer geringen Problemkomplexität (lineare Zusammenhänge, wenige Input-Parameter) reicht für die Bestimmung der Regressionskoeffizienten häufig eine kleinere Anzahl an Versuchspunkten aus. Im Vergleich hierzu fordern beispielsweise künstliche neuronale Netze, abhängig von der Wahl an Neuronen, bereits bei geringer Komplexität eine steigende Anzahl an benötigten Trainingsdaten. [9, 25]

\subsection{Methoden zur Zielgrößenoptimierung betrachteter Clinchpunkteigenschaften}

Bei der gleichzeitigen Maximierung der Halsdicke und des Hinterschnitts, abhängig von Werkzeug- beziehungsweise Prozessparametern, handelt es sich um ein mehrkriterielles Optimierungsproblem. Die zur Lösung dessen verwendeten genetischen Algorithmen (unter anderem Wang, X. et al. (2018), Israel (2015) und Drossel et al. (2013)) zeigten ein hohes Potential bei der Ableitung optimaler Parameterkombinationen. Speziell die Erzeugung von Pareto-Fronten ermöglicht hierbei die Identifizierung von bestmöglichen Lösungen, bei welchen es nicht mehr möglich ist, eine der beiden Zielgrößen zu verbessern, ohne zeitgleich die Verschlechterung der Anderen hinnehmen zu müssen. Somit bietet diese Vorgehensweise eine sehr gute Option zur Erzeugung eines Lösungsraumes, aus welchem der Konstrukteur frei zwischen unterschiedlichen optimierten Zielgrößenkombinationen wählen kann.

Eine weitere vielversprechende Auslegungsmethode von Clinchverbindungen zeigte die direkte Kopplung von FE-Simulationen mit einem genetischen Algorithmus. Beispielsweise erzielte die Beschreibung der Werkzeugkontur mittels Bézier-Kurven (Wang, M. et al. (2018)) und die direkte Übertragung von Designvariablen zwischen FE-Modell und genetischem Algorithmus eine iterative Identifizierung wichtiger Konturbereiche und die stetige Optimierung dieser. Resultierend konnte eine optimierte Gestaltung der Werkzeugkontur (Matrize) unabhängig zuvor ausgewählter Geometrieparameter erzielt werden.

\section{Zusammenfassung und Ausblick}

Im Kontext der Verbesserung von mechanischen Fügeverbindungen hat der vorliegende Beitrag, exemplarisch für das Clinchverfahren, Potentiale für den Einsatz datengestützter Methoden vorgestellt. Der Fokus lag auf der Identifizierung vorhandener Forschungsarbeiten und der analytischen Betrachtung der Vorgehensweisen zur Wissensentdeckung in Datenbanken und der daraus resultierenden datengestützten Optimierung relevanter Auslegungskriterien. Aufbauend auf einer systematischen Literaturrecherche konnten beginnend 13 Beiträge ermittelt und anschließend ausgewertet werden. Die Überführung der erworbenen Erkenntnisse in eine Methoden-Matrix bietet dabei eine übersichtliche Darstellung sämtlicher Vorgehensweisen. Zusammenfassend zeigten sich vielversprechende Resultate für die Gestaltung und Auslegung optimierter Clinchpunkteigenschaften. Im Gegensatz zu kostenintensiven physikalischen Experimenten, ermöglichen die datengestützten Vorgehensweisen eine effiziente Verbesserung der untersuchten Kenngrößen und die damit verbundene Optimierung des Bauteilverhaltens (Zug- und Scherfestigkeit). Kritisch ist jedoch der teilweise nur sehr eingeschränkte Parameterumfang mit dem überwiegenden Ziel zur Optimierung der Werkzeugkonturen (Stempel und Matrize) zu betrachten. Die Einbeziehung von Prozessparametern wird hierbei nur sehr begrenzt realisiert. Ebenfalls ist die Betrachtung weiterer maschineller Lernprozesse (Entscheidungsbäume, Support Vector Machines etc.) hinsichtlich derer Eignung zur optimierten Gestaltung von Clinchverbindungen nach aktuellem Stand nicht bekannt und wird Bestandteil nachfolgender Forschungsarbeiten. Speziell der erneute Aufbau eines künstlichen neuronalen Netzes auf Basis eines umfangreichen Datensatzes in Kombination mit einer Pareto-Optimierung könnte zu einer weiteren Steigerung der Clinchpunkteigenschaften führen. 
Tabelle 2: Methoden-Matrix für die datengestützte Analyse und Optimierung von Clinchverbindungen

\begin{tabular}{|c|c|c|c|c|c|c|c|c|c|c|c|}
\hline 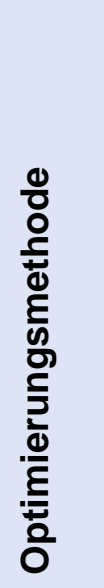 & 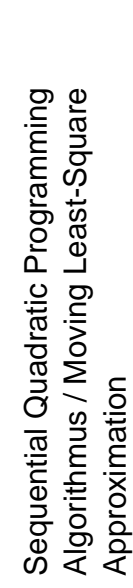 & 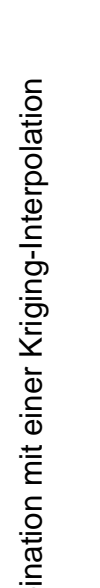 & 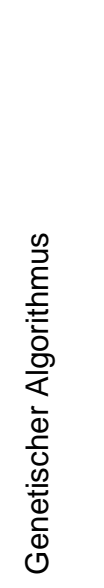 & 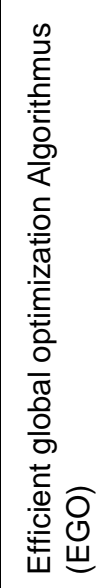 & 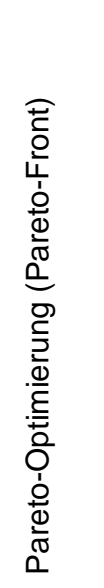 & 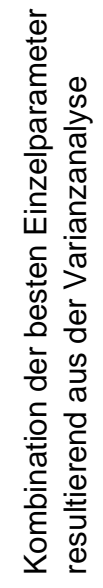 & 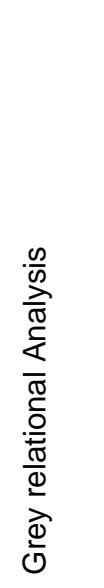 & 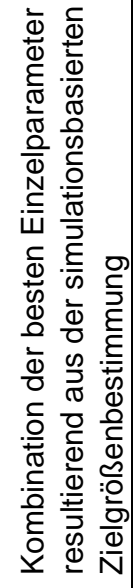 & 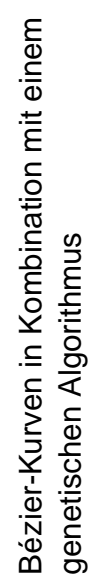 & 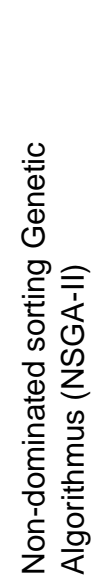 & 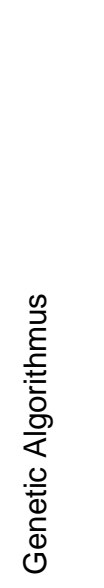 \\
\hline 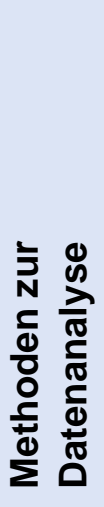 & 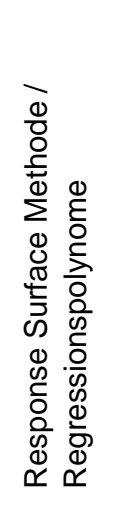 & 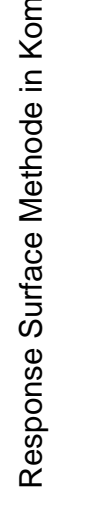 & 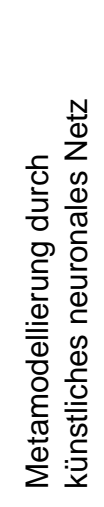 & 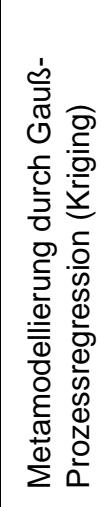 & 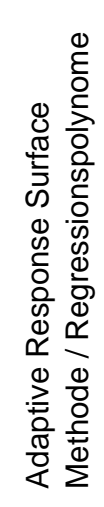 & 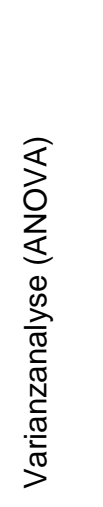 & 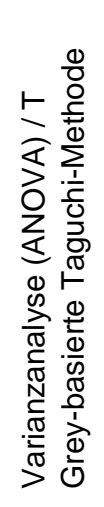 & 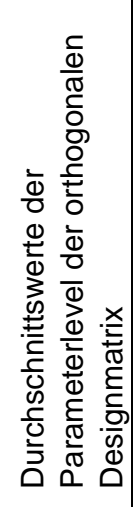 & 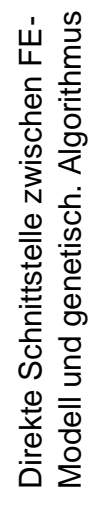 & 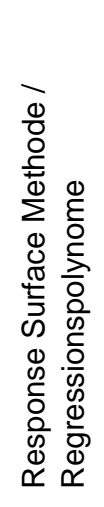 & 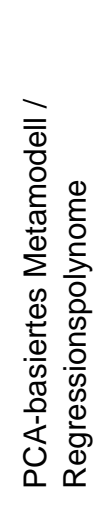 \\
\hline 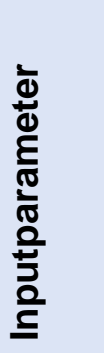 & 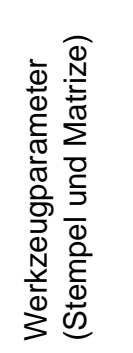 & 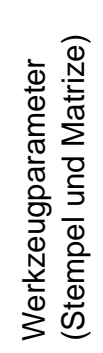 & 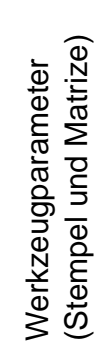 & 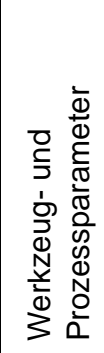 & 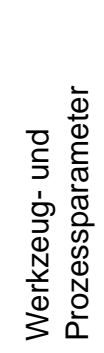 & 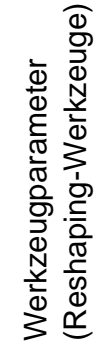 & 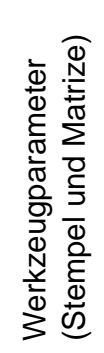 & 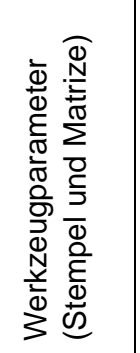 & 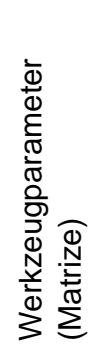 & 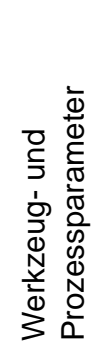 & 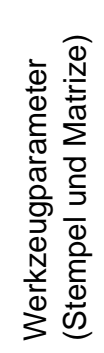 \\
\hline 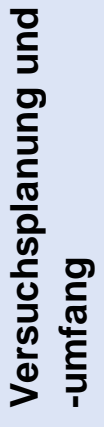 & 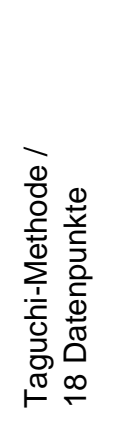 & 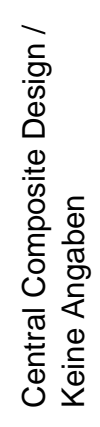 & 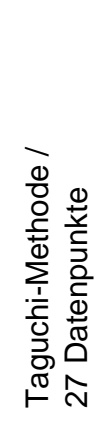 & 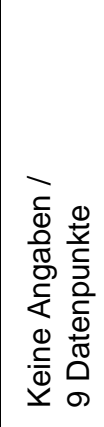 & 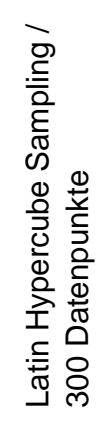 & 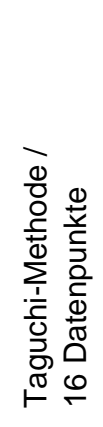 & 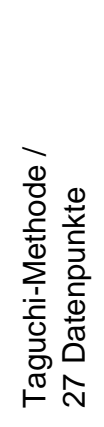 & 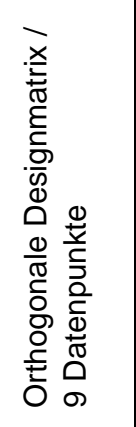 & 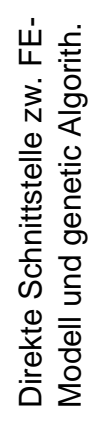 & 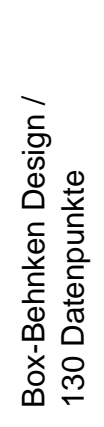 & 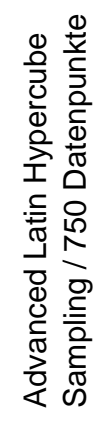 \\
\hline 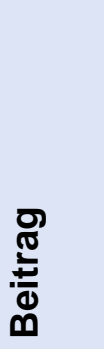 & 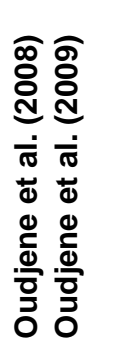 & 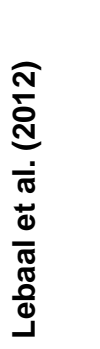 & 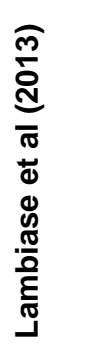 & 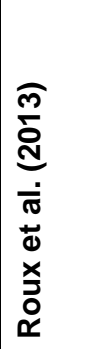 & 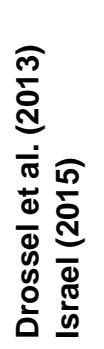 & 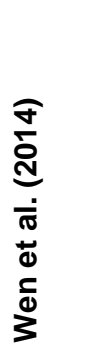 & 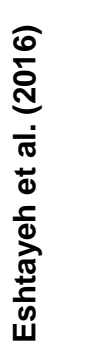 & 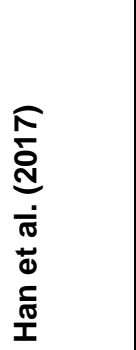 & 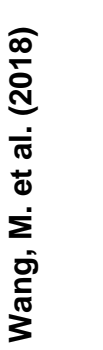 & 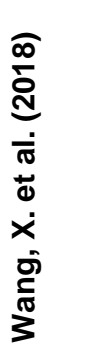 & 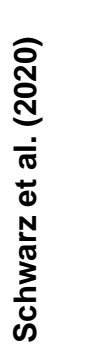 \\
\hline
\end{tabular}




\section{Danksagung}

Funded by the Deutsche Forschungsgemeinschaft (DFG, German Research Foundation) TRR 285 - Project-ID 418701707

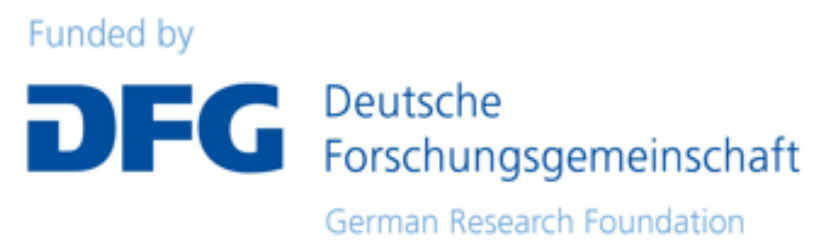

\section{Literaturverzeichnis}

[1] Ostermann, F.: Anwendungstechnologie Aluminium. Berlin Heidelberg: Springer Vieweg, 2014.

[2] Schuler, V. et al.: Schweißtechnisches Konstruieren und Fertigen. Berlin: Springer-Verlag, 2013.

[3] Neugebauer, R. et al.: Entwicklung eines Konstruktionssystems für den rechnerischen Festigkeitsnachweis von punktförmig mechanisch gefügten Bauteilen. Hannover: EFB-Forschungsbericht 323. 2010.

[4] Wittel, H., et al.: Roloff/Matek Maschinenelemente. Normung, Berechnung, Gestaltung. Wiesbaden: Springer Vieweg, 2017.

[5] Voelkner, W. et al.: Fügen von Feinblechen mittels Durchsetzfügen-Kleben und Stanznieten-Kleben. EFBForschungsbericht Nr.102. Hannover: Europäische Forschungsgesellschaft für Blechverarbeitung e.V., 1997.

[6] Fahrenwaldt, H. J. et al.: Fügen durch Umformen. In: Praxiswissen Schweißtechnik. Wiesbaden: Springer Vieweg, 2014.

[7] N.N.: Firmeninformationen der TOX Pressotechnik GmbH \& Co. KG., Weingarten.

[8] Deutscher Verband für Schweißen und verwandte Verfahren: Taschenbuch DVS-Merkblätter und Richtlinien - Mechanisches Fügen, Fachbuchreihe Schweißtechnik. Düsseldorf: DVS-Media GmbH, 2009.

[9] Witten, I. H., et al.: Data Mining. Practical Machine Learning Tools and Techniques. Cambridge: Morgen Kaufmann, 2017.

[10] Vajna S., et al.: Wissensverarbeitung. In: CAx für Ingenieure. Berlin, Heidelberg: Springer Vieweg, 2018.

[11] Oudjene, M., et al.: Geometrical optimization of clinch forming process using the response surface method. AIP Conference Proceedings 908, 2007.

[12] Oudjene, M., et al.: Shape optimization of clinching tools using the response surface methodology with Moving Least-Square approximation. Journal of Materials Processing Technology 209, 2009.

[13] Lebaal, N., et al.: The optimal design of sheet metal forming processes: application to the clinching of thin sheets. International Journal of Computer Applications in Technology 43, 2012.

[14] Lambiase, F., et al.: Optimization of the clinching tools by means of integrated FE modeling and artificial intelligence techniques. Procedia CIRP 12, 2013.

[15] Roux, E., et al.: Kriging metamodel global optimization of clinching joining processes accounting for ductile damage. Journal of Materials Processing Technology 213, 2013.

[16] Drossel, W. et al.: Sensititvitätsanalyse und Robustheitsbewertung beim mechanischen Fügen. Hannover: EFB-Forschungsbericht 376. 2013.

[17] Israel, M.: Sensitivitäts- und Robustheitsanalyse beim Clinchen dicker Stahlbleche. Dissertation. Chemnitz: Fraunhofer-Institut für Werkzeugmaschinen und Umformtechnik IWU, 2015.

[18] Wen, T., et al.: On a reshaping method of clinching joints to reduce the protrusion height. International Journal of Advanced Manufacturing Technology 71, 2014.

[19] Eshtayeh, M., et al.: Multi objective optimization of clinching joints quality using Grey-based Taguchi method. International Journal of Advanced Manufacturing Technology 87, 2016.

[20] Han, X., et al.: Optimization of geometrical design of clinching tools in clinching process with extensible dies. Journal of Mechanical Engineering Science 231, 2017.

[21] Wang, M., et al.: Shape optimization methodology of clinching tools based on Bezier curve. International Journal of Advanced Manufacturing Technology 94, 2018.

[22] Wang, X., et al.: Finite element simulation on investigations, modeling, and mulitobjective optimization for clinch joining process design accounting for process parameters and design constraints. International Journal of Advanced Manufacturing Technology 96, 2018.

[23] Schwarz, C., et al.: Optimization of thick sheet clinching tools using principal component analysis. International Journal of Advanced Manufacturing Technology 106, 2020.

[24] DVS e. V.: EFB e. V.: Merkblatt DVS/EFB 3420-2. Clinchen -Überblick. Düsseldorf: DVS-Media GmbH, 2012.

[25] Siebertz, K., et al.: Statistische Versuchsplanung. Design of Experiments (DoE). Berlin, Heidelberg: Springer Vieweg, 2017. 